\title{
ON CONTINUITY OF INVARIANT MEASURES
}

\author{
ANDREW ADLER
}

\begin{abstract}
Main TheORem. Let $\Phi$ be a set of transformations on a set $X$. The following conditions are then equivalent:

(1) There is a noncontinuous finitely additive measure defined on all subsets of $X$ and invariant under all transformations in $\Phi$.

(2) There is an integer $m$ such that for any finite subset $F$ of $\Phi$ there is a finite subset $A_{F}$ of $X$, with no more than melements, such that each $f$ in $F$ acts as a permutation on $A_{F}$.
\end{abstract}

1. Introduction. Let $X$ be a nonempty set, and let $\mathscr{A}$ be an algebra of subsets of $X$. By a measure $\mu$ on $(X, \mathscr{A})$ we mean a finitely additive nonnegative set function on $\mathscr{A}$ such that $\mu(X)=1$. Let $\Phi$ be a family of functions from $X$ to $X$ such that for any $f$ in $\Phi$ and any $U$ in $\mathscr{A}, f^{-1} U$ is in $\mathscr{A}$ (that is, the algebra $\mathscr{A}$ is invariant under $\Phi$ ). The measure $\mu$ is $\Phi$-invariant if $\mu\left(f^{-1} U\right)=\mu(U)$ for any $f$ in $\Phi$ and any $U$ in $\mathscr{A}$. If $V$ is in $\mathscr{A}$, the range of $\mu$ on $V$ is the set of all numbers $\mu(U)$, where $U$ ranges over all subsets of $V$ in $\mathscr{A}$. In this paper we find some conditions on $\Phi$, $\mathscr{A}$ that ensure that for any $\Phi$-invariant measure $\mu$ on $\mathscr{A}$ and any $V$ in $\mathscr{A}, \mu$ has full range $[0, \mu(V)]$ on $V$. A complete characterization is reached in the case that $\mathscr{A}$ is the algebra of all subsets of $X$. There are only partial results for the general case.

There are a number of papers that deal with special cases of our problem. They look at the situation in which $X$ is a topological semigroup, $\mathscr{A}$ is the algebra of Borel subsets of $X$, and $\Phi$ is $X$ (acting on itself by left multiplication). Granirer in [4] obtained an almost complete answer for discrete semigroups. A remaining problem was settled by Chou in [3]. Recently, Snell has in [6] extended the results of Granirer and Chou to locally compact groups. By looking at the problem in a more general setting, we are able to extend these various results and, to some degree, to simplify their proofs.

2. Some preliminary results about $\Phi$-invariant measures. The measure $\mu$ is said to be continuous if for every $\varepsilon>0$, there exists a finite partition $X=X_{1} \cup \cdots \cup X_{n}$ of $X$, with the $X_{i}$ in $\mathscr{A}$, such that $\mu\left(X_{i}\right)<\varepsilon$ for all $i$.

Received by the editors February 26, 1973.

AMS (MOS) subject classifications (1970). Primary 43A07.

Key words and phrases. Finitely additive, invariant, measure, continuous, ultrafilter.

(c) American Mathematical Society 1973 
Lemma 1 (SobCZyK AND HAMmer [7]). Let $\mu$ be a continuous measure on $(X, \mathscr{A})$. Then there is a dense subset $S$ of $[0,1]$, and a collection $\left\{U_{s}\right\}$ of elements of $\mathscr{A}$, where $s$ ranges over $S$ such that (i) if $s<t, U_{s} \subset U_{t}$, and (ii) $\mu\left(U_{s}\right)=s$ for all $s$ in $S$. If $\mathscr{A}$ is a $\sigma$-algebra, $S$ may be taken to be all of $[0,1]$.

So if $\mu$ is continuous, and $\mathscr{A}$ is a $\sigma$-algebra, then $\mu$ attains full range, and moreover does so on a nested collection of sets.

Lemma 2 (SobczyK AND Hammer [7]). Any measure $\mu$ has a unique decomposition (apart from the order of the terms) in the form $\mu=\kappa+$ $\sum_{i=1}^{\infty} a_{i} \lambda_{i}$, where $\kappa$ is continuous, the $a_{i}$ are nonnegative, and the $\lambda_{i}$ are $\{0,1\}$-valued finitely independent measures.

For any measure $\mu$, and any $f: X \rightarrow X$ under which $\mathscr{A}$ is invariant, define $f \mu$ by $(f \mu)(U)=\mu\left(f^{-1} U\right)$. Then $f \mu$ is a measure on $(X, \mathscr{A})$. If $\mu$ is $\{0,1\}$-valued, so is $f \mu$. If $\mu=\sum_{i=1}^{\infty} a_{i} \mu_{i}$, then $f \mu=\sum_{i=1}^{\infty} a_{i}\left(f \mu_{i}\right)$. Verification of these facts is straightforward.

Let $\mu=\kappa+\sum_{i=1}^{\infty} a_{i} \lambda_{i}$ be the Sobczyk-Hammer decomposition of $\mu$, where we choose $a_{i} \geqq a_{i+1}$ for all $i$. If $\mu$ is $f$-invariant, then $\mu=f \mu=f \kappa+$ $\sum_{i=1}^{\infty} a_{i}\left(f \lambda_{i}\right)$. Let $\lambda=\sum_{i=1}^{\infty} a_{i} \lambda_{i}$. Now $f \lambda$ is a linear combination of $\{0,1\}-$ valued measures, and $(f \lambda)(X)=\lambda(X)$. By the uniqueness of the SobczykHammer decomposition, we have $f \lambda=\lambda$, and so $f \kappa=\kappa$. If $\mu$ is not continuous, $\mu \neq \kappa$, and so not all the $a_{i}$ are zero. Let $n$ be the integer such that $a_{1}=a_{2}=\cdots=a_{n}$ but $a_{n+1}<a_{n}$ (recall the $a_{i}$ are nonincreasing). Again from the uniqueness of the decomposition, we see that $f \lambda_{i}=\lambda \pi(i)$ for $1 \leqq i \leqq n$, where $\pi$ is some permutation of $\{1, \cdots, n\}$. Hence the measure $n^{-1}\left(\lambda_{1}+\cdots+\lambda_{n}\right)$ is $f$-invariant. The argument is independent of the particular $f$ selected. So we have proved:

LeMma 3. If $(X, \mathscr{A})$ admits a noncontinuous $\Phi$-invariant measure, then $(X, \mathscr{A})$ admits a $\Phi$-invariant measure of form $1 / n\left(\lambda_{1}+\cdots+\lambda_{n}\right)$, where the $\lambda_{i}$ are $\{0,1\}$-valued independent measures.

Lemma 3 gives a substantial reduction of the original problem. It yields, for instance, a simplified proof of the following recent result:

THEOREM (SNeLl [6]). Let $S$ be an infinite subsemigroup of a locally compact topological group. Let $\mathscr{A}$ be the algebra of Borel'sets of $S$, and let $\Phi$ be $S$ acting on itself by left multiplication. Then every $\Phi$-invariant measure on $(S, \mathscr{A})$ attains range $[0,1]$ on a nested collection of sets.

Proof. By Lemma 1 and Lemma 3 , it is sufficient to show that $(S, \mathscr{A})$ does not admit a $\Phi$-invariant measure of form $n^{-1}\left(\lambda_{1}+\cdots+\lambda_{n}\right)$, where the $\lambda_{i}$ are $\{0,1\}$-valued. But this follows at once from Theorem 3 of [5]. The argument actually shows every Baire measure is already continuous. 
The next lemma is a localization result that enables one to go from infinite sets of functions to finite sets.

Lemma 4. If $(X, \mathscr{A})$ admits an $F$-invariant measure $\mu_{F}$ for every finite subset $F$ of $\Phi$, then $(X, \mathscr{A})$ admits a $\Phi$-invariant measure $\mu$. Moreover, if each $\mu_{F}$ takes on no more than $m+1$ values on subsets of $X, \mu$ can be chosen with the same property.

Proof. A technique from nonstandard analysis is used to take the appropriate limit. More detail about the ideas involved can be found for instance in [1]. Let $I$ be the collection of finite subsets of $\Phi$. For any $f$ in $\Phi$, let $K_{f}$ be the set of $F$ in $I$ such that $f$ is in $F$. The family $\left\{K_{f}\right\}$ has the finite intersection property. Let $D$ be an ultrafilter on $I$ that extends this family. For any $U$ in $\mathscr{A}$, define $\mu_{U}: I \rightarrow R$ by $\mu_{U}(F)=\mu_{F}(U)$. Define a measure $\mu$ on $(X, \mathscr{A})$ by $\mu(U)=s t\left(\mu_{U} / D\right)$, where st is the ordinary standard part function of nonstandard analysis. For any $U$ and any $F$, $0 \leqq \mu_{U}(F) \leqq 1$, so the standard part of $\mu_{U} / D$ exists. It is easy to verify $\mu$ is a measure. For any $f$ in $\Phi,\left\{F: \mu_{F}(U)=\mu_{F}\left(f^{-1} U\right)\right\} \supseteq K_{f}$, and hence lies in $D$. Therefore $\mu_{U} / D=\mu_{f^{-1} U} / D$, and so $\mu$ is $\Phi$-invariant. Since $D$ is an ultrafilter, for any partition of $I$ into $m+1$ sets, one of the sets must lie in $D$. So if each $\mu_{F}$ takes on no more than $m+1$ values, the same is true of $\mu$.

3. Analysis of the discrete case. In this section, $\mathscr{A}$ will be the algebra of all subsets of $X$. We characterize those $\Phi$ for which there exists a $\Phi$ invariant discontinuous measure. The main additional tool is the following:

LEMma 5. Let $\lambda$ be a $\{0,1\}$-valued measure on the collection of all subsets of $X$, and let $f: X \rightarrow X$ be such that $f \lambda=\lambda$. Then $f$ is the identity map on a set of $\lambda$-measure 1 .

Proof. The result has been known for a fairly long time, having been proved by (among others) Keisler and M. Rudin. A proof appears in print in [2] for countable $X$. That proof works equally well for arbitrary $X$.

THEOREM. The following are equivalent conditions on $\Phi$ :

(1) $X$ supports a $\Phi$-invariant noncontinuous measure.

(2) There is an integer $m$ such that for any finite subset $F$ of $\Phi$ there is a subset $A_{F}$ of $X$, with no more than $m$ elements, such that each $f$ in $F$ acts as a permutation on $A_{F}$.

Proof. We first show that if (2) holds, so does (1). Define measure $\mu_{F}$ on $X$ by assigning equal nonzero mass to the objects of $A_{F}$ and normalizing so that $\mu_{F}\left(A_{F}\right)=1$. Since each $f$ in $F$ permutes $A_{F}, \mu_{F}$ is $F$ invariant on $X$, and takes on no more than $m+1$ values. By Lemma 4, 
there is then a $\Phi$-invariant measure on $X$ taking on no more than $m+1$ values.

Next we show that if (1) holds, so does (2). If (1) holds, then by Lemma $3 X$ supports a $\Phi$-invariant measure of form $\mu=n^{-1}\left(\lambda_{1}+\cdots+\lambda_{n}\right)$, where the $\lambda_{i}$ are $\{0,1\}$-valued. If $\mu$ is $f$-invariant and $g$-invariant, it is invariant under the composition $f \circ g$ of $f$ and $g$. So we may assume $\Phi$ is a semigroup. We may further assume that $\Phi$ contains the identity map $e$.

Each $f$ in $\Phi$ induces a permutation on the $\lambda_{i}$. Put $f \sim g$ if $f \lambda_{i}=g \lambda_{i}$ for $1 \leqq i \leqq n$. The relation $\sim$ is a congruence on the semigroup $\Phi$. The equivalence classes under the natural multiplication form a group $G$ isomorphic to a subgroup of the permutation group on $n$ letters. Let $m$ be the order of $G$.

We need to characterize the relation $\sim$ more closely. If $f$ and $g$ agree on a set of $\mu$-measure 1 , then clearly $f \sim g$. The converse also holds. If $f \sim e$, then $f \lambda_{i}=e \lambda_{i}=\lambda_{i}$ for $1 \leqq i \leqq n$. Hence by Lemma $5, f$ is the identity on a set of $\lambda_{i}$-measure 1 for all $i$, and so $f$ is the identity on a set of $\mu$-measure 1 . In general, if $f \sim g$, then since $G$ is a group, $h f \sim h g \sim e$ for some $h$. But then $h$ must be one-to-one on a set of $\mu$-measure 1 . It follows that $f=g$ on a set of $\mu$-measure 1 .

Now let $F$ be a finite subset of $\Phi$. The relation $\sim$ partitions $F$ into sets $F_{1}, \cdots, F_{m}$. It is convenient here to assume that each element of $G$ is represented in $F$. There exists then a set $U$ of $\mu$-measure 1 such that

(i) If $f, g$ are in $F_{i}$, then $f$ and $g$ agree on $U$.

(ii) If $f$ is in $F_{i}$ and $g$ is in $F_{j}$, where $i \neq j$, then $f$ and $g$ differ at every point of $U$.

This follows from our characterization of the relation $\sim$ and from the fact that the intersection of a finite number of sets of measure 1 has measure 1 .

Write down now all true relations of the form $f \circ g \sim h$, where $f, g, h$ range over $F$. There is only a finite number of these. Therefore there is a set $V$ of $\mu$-measure 1 such that for any $x$ in $V$, and any relation $f \circ g \sim h$, $(f \circ g) x=h(x)$.

So $U \cap V$ has $\mu$-measure 1 , and so there is a point $x$ in it. Let $A_{F}$ be the set of points of form $f x$, where $f$ is in $F$. By (i) and (ii), $A_{F}$ has precisely $m$ elements. It remains to show that each element of $F$ permutes $A_{F}$.

We show that if $f$ is in $F$, and $y$ is in $A_{F}$, then $f y$ is in $A_{F}$. Now $y=g x$ for some $g$ in $F$, and so $f y=(f \circ g) x$. But since $F$ contains a representative for each element of $G, f \circ g \sim h$ for some $h$ in $F$. From our choice for $V$, $(f \circ g) x=h x$, which is in $A_{F}$. If $y$ and $z$ are in $A_{F}$ and $y \neq z, f y \neq f z$. For let $y=g x, z=h x$. $f$ and $g$ cannot be equivalent. So $f \circ g$ and $f \circ h$ are not equivalent, and so $(f \circ g) x \neq(f \circ h) x$. This completes the proof. 
4. Some remarks about the general case. Let $X$ be a compact Hausdorff space (or more generally an $H$-closed space). Let $\mathscr{A}$ be the algebra generated by the open sets, and let $\Phi$ be a family of continuous functions on $X$. Suppose $(X, \mathscr{A})$ admits a discontinuous $\Phi$-invariant measure. By Lemma $3,(X, \mathscr{A})$ admits a $\Phi$-invariant measure of form

$$
\mu=n^{-1}\left(\lambda_{1}+\cdots+\lambda_{n}\right) \text {, }
$$

where the $\lambda_{i}$ are $\{0,1\}$-valued measures (ultrafilters) on it. Let $p_{i}(1 \leqq i \leqq n)$ be the limit points of the $\lambda_{i}$. Each $f$ in $\Phi$ permutes the $\lambda_{i}$, and so since $f$ is continuous it permutes the $p_{i}$. Conversely, if there is a finite set $P$ such that each $f$ in $F$ permutes $P,(X, \mathscr{A})$ admits a discontinuous $\Phi$-invariant measure. This result is not nearly as informative as the results for the discrete case. The ideas used in $\$ 3$ can be used to give a construction of all $\Phi$-invariant measures that take on a finite number of values. We cannot give such a general construction in compact spaces.

The main obstruction to deeper knowledge is our failure to prove an analogue of Lemma 5 in a more general setting. Lemma 5 fails in some compact Hausdorff spaces (the one point compactification of the discrete space of cardinality $\omega_{1}$ provides an example). We conjecture Lemma 5 holds for locally compact metric spaces and continuous maps. We have verified it does hold in a number of cases, the most interesting one being the real line and continuous monotone maps.

\section{REFERENCES}

1. A. Adler, Invariant means via the ultrapower, Math. Ann. 202 (1973), 71-76.

2. D. Booth, Ultrafilters on a countable set, Ann. Math. Logic 2 (1970), 1-24. MR 43 \#3104.

3. C. Chou, On a conjecture of $E$. Granirer concerning the range of an invariant mean, Proc. Amer. Math. Soc. 26 (1970), 105-107. MR 41 \#5519.

4. E. Granirer, On the range of an invariant mean, Trans. Amer. Math. Soc. 125 (1966), 384-394. MR 34 \#4390.

5. E. Granirer and A. Lau, Invariant means on locally compact groups, Illinois J. Math. 15 (1971), 249-257. MR 43 \#3400.

6. R. Snell, The range of invariant means on topological groups and semigroups, Proc. Amer. Math. Soc. (to appear).

7. A. Sobczyk and P. Hammer, A decomposition of additive set functions, Duke Math. J. 11 (1944), 839-846. MR 6, 129.

Department of Mathematics, University of British Columbia, Vancouver 8, Brtish Columbia, Canada 